\title{
Cytomegalovirus-esophagitis as initial presentation of HIV infection mimicking adenocarcinoma
}

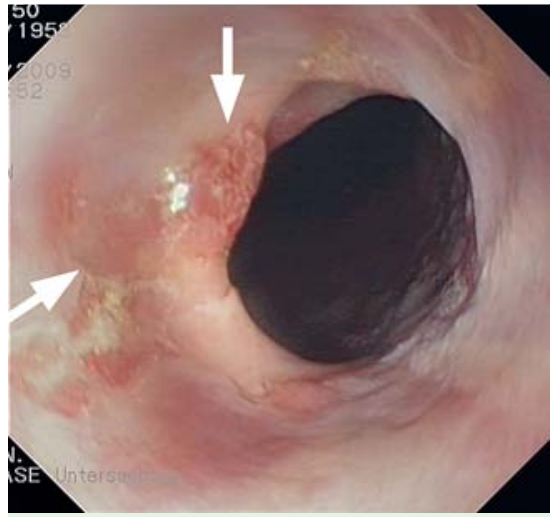

Fig. 1 Edge (left arrow) and ulcerated field (right arrow) of a tumor mass.

A 49-year-old man presented with dysphagia and weight loss of $5 \mathrm{~kg}$ within 2 months. An esophagogastroduodenoscopy (EGD) was performed in a community hospital. An ulcerated tumor of the cardia was seen and a biopsy was interpreted as a well-differentiated adenocarcinoma. The patient was referred to our hospital to complete the staging and initiate surgical intervention. The EGD was repeated and the previously described cardiac tumor was confirmed ( $\bullet$ Fig. 1 ).

An endoscopic ultrasound (EUS) revealed a $30 \times 8 \mathrm{~mm}$ tumor ( $\bullet$ Fig. 2 ).

The mucosal and submucosal layers could not be delineated but the muscular layer was intact. The macroscopic view resulted in the diagnosis of an early gastric cancer $\mathrm{uT} 1 \mathrm{sm}$ and consequently surgical management was considered. In addition, the histologic examination revealed a damaged mucosal architecture and epithelial cells with atypia. In parallel, the medical history revealed homosexual preferences of the patient; HIV-testing was initiated. The viral load was $464000 /$ copies/mL and CD4

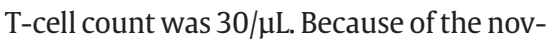
el aspect of an immunosuppressive state, the diagnosis of cytomegalovirus (CMV)gastroesophagitis was considered, and we requested the biopsies from the local pathology center for re-evaluation. Immunhistochemical examination of all biopsies indicated the presence of multiple CMV pp65-positive cells ( $\bullet$ Fig. 3).

The most common presentation of CMVesophagitis are well-circumscribed ulcerations, which can differ in number, size,

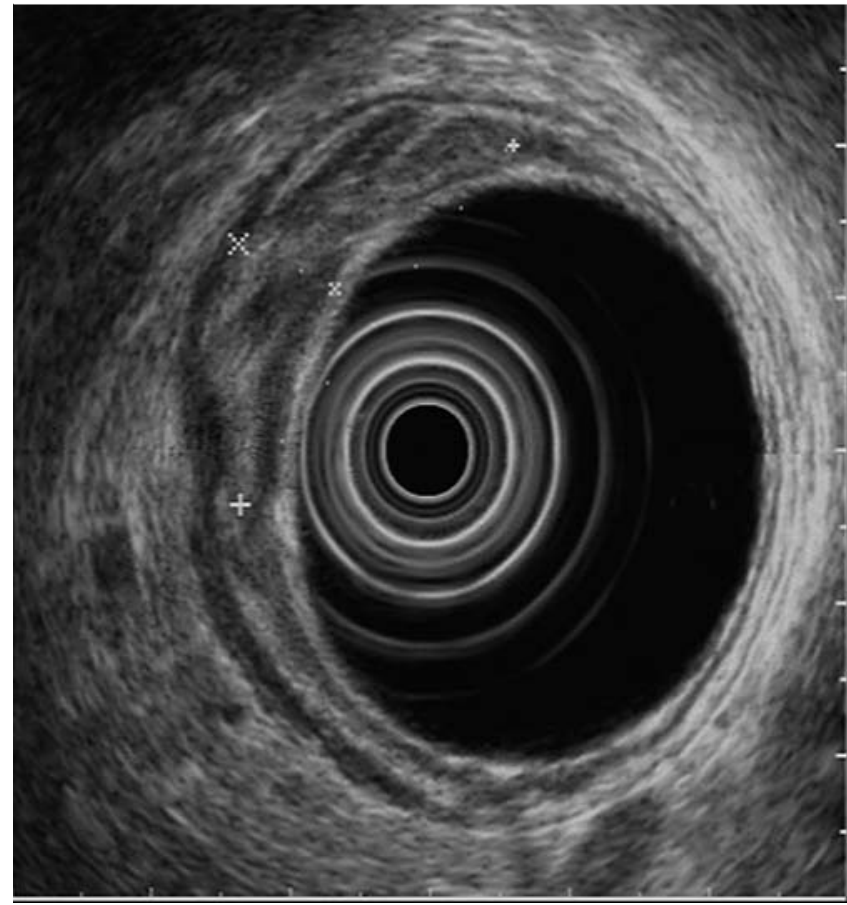

Fig. 2 Endoscopic ultrasound showed an intact muscular layer.

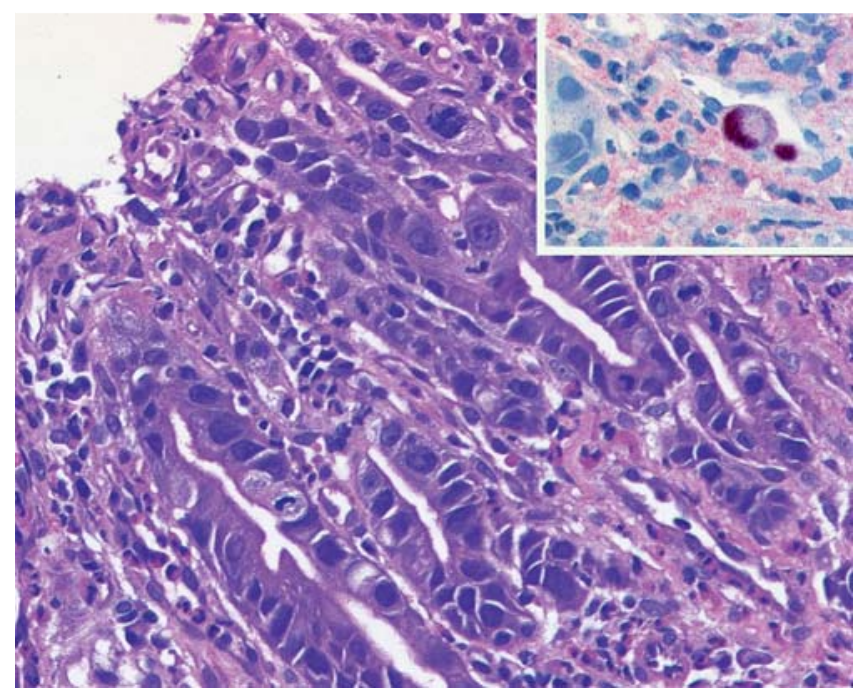

Fig. 3 Histologic examination and immunohistochemical staining showed cytomegalovirus-positive cells.

and depth [1]. Although the appearance is endoscopically highly variable, an ulcerated, bulged, and irregularly circumscribed tumor is a rare condition. The surgical intervention was delayed and treatment with ganciclovir was initiated. After CMV therapy EGD and EUS were repeated. Only a small ulcer of the cardia remained and EUS was normal. The histologic investigation revealed inflammation and mild regenerative features ( $\bullet$ Fig. 4).
In summary this case demonstrates the difficulties in differentiating between an inflammatory process and an invasive tumor by endoscopy, ultrasound, and histology [2,3], and underlines the importance of a detailed medical history. By re-evaluating the diagnosis, the patient was saved from an esophagocardiac resection.

Endoscopy_UCTN_Code_CCL_1AB_2AC_3AZ 


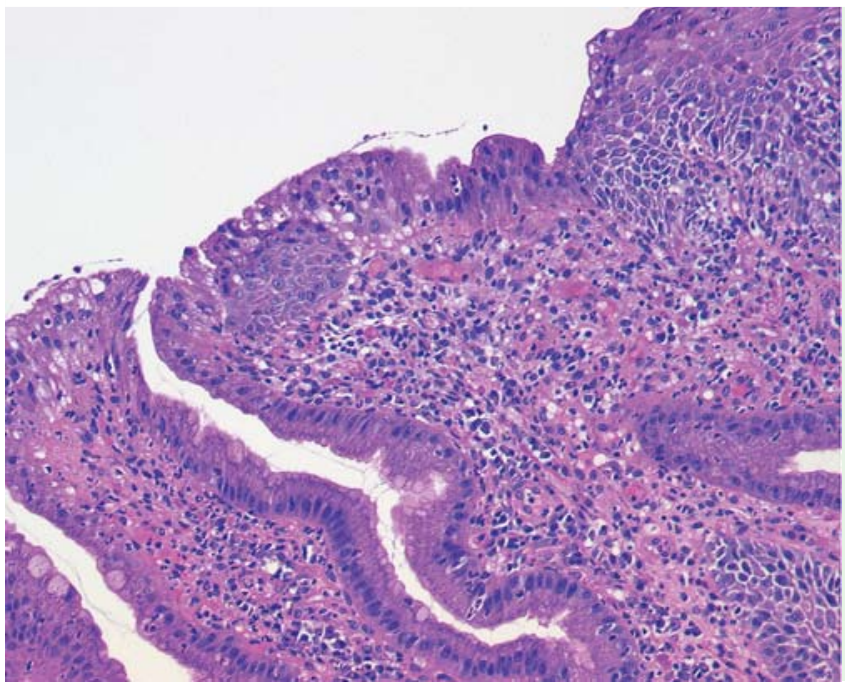

Fig. 4 Regeneration of cytoarchitecture after treatment for cytomegalovirus.

G. Weinberg ${ }^{1}$, B. Siegmund ${ }^{2}$, M. Grünbaum ${ }^{3}$, C. Loddenkemper ${ }^{3}$, T. Schneider ${ }^{4}$

1 Department of Nephrology and Intensive Care Medicine, Charite Campus Virchow Klinikum, Berlin, Germany

2 Department of Gastroenterology, Charite Campus Benjamin Franklin, Berlin, Germany

\section{References}

1 Wilcox CM, Straub RF, Schwartz DA. Prospective endoscopic characterization of cytomegalovirus esophagitis in AIDS. Gastrointest Endosc 1994; 40: 481 - 484

2 Neumann H, Fry L, Wilcox C et al. Gastrointestinal imaging in HIV. J HIV Ther 2008; 13: $58-64$

3 Singh SP, Odze RD. Multinucleated epithelial giant cell changes in esophagitis: a clinicopathologic study of 14 cases. Am J Surg Pathol 1998; 22: 93 -99

Bibliography

DOI $10.1055 / \mathrm{s}-0029-1215352$

Endoscopy 2010; 42: E22 -E23

(c) Georg Thieme Verlag KG Stuttgart · New York . ISSN 0013-726X

Corresponding author

G. Weinberg, MD

Department of Nephrology and Intensive Care Medicine

Charite Universitätsmedizin Berlin

Campus Virchow Klinikum

Augustenburger Platz 1

13353 Berlin

Germany

Fax: +49-30-450565954

gordon.weinberg@charite.de 